\title{
LA POLYARTHRITE MASCULINE
}

\author{
PAR \\ J. FORESTIER et F. JACQUELINE \\ Aix-les-Bains, France
}

(RECEIVED FOR PUBLICATION SEPTEMBER 22, 1952)

Les aspects cliniques et radiologiques de la polyarthrite chronique évolutive sont multiples. Ce fait semble être en partie sous l'influence du processus inflammatoire qui peut diminuer d'intensité ou disparaître à n'importe quelle phase de la maladie; mais une étude de cas masculins permet de soupçonner que l'âge, c'est à dire un des facteurs essentiels du terrain, joue un rôle dans la détermination du type d'altérations radiologiques.

L'élément inflammatoire de la polyarthrite est, classiquement, la prolifération synoviale; l'altération radiologique à un stade avancé est l'atrophie ou la destruction des extrémités osseuses. Chez l'homme, cet élément inflammatoire peut revêtir plusieurs aspects: proliférations, épanchements plus ou moins abondants, ou épaississements durs. Il nous a paru intéressant de rechercher s'il y avait une relation entre l'élément clinique constaté et le type d'altérations radiologiques.

\section{Matériel}

Notre étude est basée sur 48 cas dont l'ancienneté est très variable (de quelques mois à 45 ans); neuf de ces cas ont été suivis pendant une période qui est pour certains de plus de 15 ans, ce qui présente un intérêt, car, il a été ainsi possible de connaître la réponse aux diverses thérapeutiques. Le nombre de nos cas est peu élevé mais nous n'avons voulu retenir que les dossiers comprenant une radiographie des articulations sacro-iliaques (pour éliminer le diagnostic de spondylarthrite) et un cliché d'une des articulations atteintes: hanche, poignet, etc. Or, comme il s'agit de malades de clientèle privée, ce sont surtout les cas sévères, avec lésions destructives qui ont été radiographiés. Nous ne pouvons donc pas présenter de pourcentage des différentes formes cliniques, car les cas à poussées fluxionnaires ou à petits épanchements qui n'entrainent qu'une invalidité soit temporaire, soit discrète, n'ont pas subi d'examen radiologique.

Fréquence.-La proportion des cas masculins et féminins est très différente. Fletcher et Rowley (1952) donnent par exemple un homme pour deux femmes; nous-mêmes avons indiqué le pourcentage de 20 pour cent. de cas masculins. Cette différence nous semble due à la définition même de la polyarthrite. Dans notre statistique nous n'avions tenu compte que des cas polyarticulaires avec $\vec{\omega}$ atteinte symétrique; nous éliminions alors en particulier les oligo-arthrites. Le diagnostic de⿳⺈. polyarthrite chez l'homme doit être fait avec plusde précautions que chez la femme en raison de la fréquence des spondylarthrites à début périphériqueĩ et de l'existence de la goutte polyarticulaire chroni- $\rightarrow$ que; de plus, il ne faut pas oublier qu'il s'agit non음 d'une affection dont l'étiologie est connue, maisd'un syndrome clinique et radiologique dont les limites sont quelquefois difficiles à définir. Toute- $\mathbb{\bigotimes}_{\mathbb{Q}}$ fois, le rhumatisme inflammatoire localisé aux articulations périphériques semble être beaucoupณ plus fréquent chez l'homme que l'on ne le pensait.

Antécédents.-Onze cas seulement présentaient

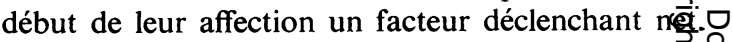
Il s'agissait le plus souvent d'antécédents infectieux; abcès dentaires, amygdalite, blennorragie (un cas).

Divers auteurs ont noté que la polyarthite de l'homme⿳ू était souvent consécutive à une gonococcie. Selon nous, $\underset{\mathbb{Q}}{\mathbb{Q}}$ il n'existe pas de véritable polyarthrite gonococcique ou blénnoragique l'infection joue selon nous un rôle deô "facteur déclenchant". De plus, nous avons noté,, lors de blennorragie, des douleurs articulaires, accom-? pagnées ou non de gonflement, localisées surtout auơ niveau des membres inférieurs, mais généralement ces manifestations sont de courte durée et ne laissent habituellement pas de séquelles; nous pourrions faire. la même remarque pour les poussées articulaires fugitives qui suivent une angine. Ces poussées réagissent rapidement aux antibiotiques.

Trois cas ont débuté aussitôt après une fracture articulaire: ce fut l'articulation traumatisée quio présenta d'abord les signes inflammatoires. UnD sujet ressentit les premiers symptômes les jours quioㅡ. suivirent une fracture du crâne. Dans un casĩ observé récemment, la polyarthrite débuta aussitôt après un ictus, qui laisse actuellement pour séquelle $\mathrm{N}^{N}$ une monoplégie.

Les poussées ultérieures sont déclenchées soit parog le même facteur, soit par d'autres. Il est à noteco que des cas de polyarthrite ayant débuté avant 20 ans et stabilisés les années suivantes ont présentée à nouveau une poussée quelquefois sévère quand 
les malades atteignirent la quarantaine. Or la plupart de nos cas de polyarthrite chronique évolutive ont débuté entre 30 et 50 ans, l'âge est donc un facteur déclenchant. Le terrain joue donc un rôle important; comme nous le verrons pluș loin, les altérations notées à partir de cet âge sont destructives.

Age de Début.-Cinq de nos cas ont débuté entre 16 et 20 ans; la plupart ont débuté entre 30 et 50 ans (28 cas), un de nos cas après 70 ans.

\section{Symptômes de Début}

\section{Premières Localisations}

Atteinte Monoarticulaire.-La maladie débuta par une atteinte monoarticulaire dans six cas. Il s'agissait d'un genou, d'un poignet, d'une épaule ou d'un coude et cette atteinte persistante est restée monoarticulaire pendant une période variant de quelques mois à 6 ans.

L'atteinte de l'épaule fut la seule localisation de début dans deux cas; ceci est à souligner, car bien souvent en présence de douleurs et de raideur de l'épaule, le diagnostic de périarthrite a été fait le plus fréquemment. Ce diagnostic de périarthrite semblait être confirmé par la constatation de signes d'arthrose cervicale, car cette association est classique; or, la plupart de nos sujets ont 40 ou 50 ans, période de la vie où il est déjà banal de trouver une raideur du cou, des affaissements discaux, et quelques ostéophytes au niveau de la région cervicale inférieure. Il faut en présence d'une périarthrite de l'épaule pratiquer une sédimentation globulaire et, si les symptômes cliniques persistent plusieurs mois, il faut examiner à nouveau soigneusement toutes les articulations et en particulier les poignets.

Dans trois autres cas, les deux épaules furent atteintes simultanément; le diagnostic de polyarthrite doit être alors envisagé dès le début car la simultanéité de deux périarthrites est exceptionnelle. La périarthrite à bascule existe, mais il faut être prudent devant un tel diagnostic.

Atteinte Oligo-Articulaire.--Le début fut oligoarticulaire dans trentę cas. Il s'agissait soit d'une atteinte symétrique, soit d'une atteinte de deux, trois ou quatre articulations non symétriques et l'affection est restée pauci-articulaire pendant de très nombreuses années (dans un de nos cas elle l'est restée pendant 31 ans) pour devenir ensuite polyarticulaire et être alors généralement symétrique. Le problème de l'oligo-arthrite sera étudié au diagnostic différentiel.

Atteinte Polyarticulaire.-Le début fut polyarticulaire dans dix de nos cas et dans la moitié de ceux-ci, il s'agissait de poussées fluxionnaires, c'est à dire de manifestations intermittentes.

Signes Généraux.-Les signes généraux de début furent nets dans 32 cas sur 48 . Il s'agissait soit de fièvre, soit d'amaigrissement, soit des deux le plus souvent. Ces signes de début existaient, que l'atteinte soit localisée ou polyarticulaire. La polyarthrite de l'homme s'accompagne donc fréquemment d'une atteinte de l'état général; par contre celui-ci n'est pratiquement jamais modifié à la phase de début de la spondylarthrite ankylosante dans les cas qui ne présentent que des symptômes sacroiliaques ou rachidiens (Jacqueline et al., 1952).

L'étude des symptômes de début met en évidence que le tableau clinique se constitue soit rapidement soit en de nombreuses années, c'est dire que l'évolution est variable et les tableaux cliniques multiples.

\section{Éléments Articulaires Inflammatoires et Altérations Radiologiques}

L'étude radiologique a été faite, d'une part d'après l'articulation ou les articulations dont les clichés étaient nécessaires pour faire un bilan orthopédique, et d'autre part d'après les poignets et les mains, et c'est essentiellement les modifications constatées au niveau de ceux-ci que nous rapportons ici. Nous indiquerons simplement les quelques particularités que nous avons pu noter au niveau des autres articulations.

Nous avons classé nos cas suivant leur âge au moment de l'observation et nous avons noté que l'élément articulaire inflammatoire et le type d'altérations radiologiques dépendent de celui-ci. Certains sujets ont débuté jeunes leur polyarthrite, mais ils n'ont été examinés par nous que lorsqu' ils avaient atteint 50 ou 60 ans, le processus inflammatoire ayant persisté ou étant réapparu après une longue période de stabilisation; les altérations radiologiques notées alors nous paraissent beaucoup plus difficiles à analyser (Fig. 3).

(1) Forme ankylosante de l'adulte jeune, sans destructions osseuses (Nous éliminons de notre étude les polyarthrites de l'enfance).-Quatre de nos cas de polyarthrite ont entre 18 et 26 ans. Le mode de début fut peu différent de celui des autres cas. L'atteinte est actuellement diffuse; dans deux cas la région cervicale est atteinte et chez l'un de ceux-ci, il existe une cyphose dorsale avec raideur importante. Bien qu'il s'agisse de cas évoluant depuis 6 ou 7 ans, nous n'avons noté, au niveau du rachis, que des altérations inter-apophysaires; il n'existe ni ponts osseux ni altérations des sacro-iliaques.

La plupart des articulations atteintes étaient très enraidies et quelques unes bloquées. Dans un cas les douleurs ne furent jamais vives bien que l'examen révèle des blocages articulaires. $\mathrm{Ce}$ fait clinique est à rapprocher de la relative indolence de nombreux cas de polyarthrite de l'adolescence et de l'enfance. Les modifications des mains sont différentes de celles de la polyarthrite habituelle. Les téguments de couleur légèrement violacée étaient froids et 
se laissaient mal déccller des plans profonds; les articulations inter-phalangiennes distáles étaient moins volumineuses mais très enraidies; le malade ne pouvait ni fermer ni étendre complètement les doigts. La palpation des articulations inter-phalangiennes révèlait un épaississement dur des tissus. Les déviations étaient discrètes.

L'atrophie musculaire, masquée en partie par l'épaississement dur de la peau, était prononcée. Dans un cas, le gonflement du poignet était manifeste, et la peau était tendue et chaude, mais nous n'avions pas cette impression "amadou" de tuméfaction molle qui caractérise la prolifération.

La radiographie des poignets bloqués nous a révélé une image d'ankylose osseuse sans destructions. Cette ankylose était soit localisée à certaines articulations du poignet soit diffuse. Fig. 1 montre que le carpe est devenu un véritable bloc osseux, mais qu'il a conservé une hauteur normale; les os du carpe et du métacarpe ont conservé des rapports normaux, il n'y a donc pas eu de destruction osseuse; il n'existe pas de zône de condensation. Sur ce cliché, nous ne notons pas de troubles de structure. Chez un autre malade, il existait une porose importante.

Les articulations inter-phalangiennes distales qui ont conservé un certain degré de mobilité présentent une légère sclérose de certaines articulations qui sont prolongées par des formations osseuses; celles-ci sont quelquefois difficile à différencier des altérations dites arthrosiques; mais il existe une porose nette.

La vitesse de sédimentation était élevée dans trois des cas; elle était normale dans le quatrième alors que la douleur articulaire était vive et que la raideur augmentait. La vitesse de sédimentation n'était donc pas en rapport avec l'état clinique; il est à noter que, dans ce cas, il n'existait pas de proliférations (ce manque de concordance entre l'état clinique et la vitesse de sédimentation a été noté aussi dans des cas de spondylarthrite). Le processus ankylosant sans destructions osseuses ne s'accompagne donc pas toujours de modifications de la vitesse de sédimentation (Jacqueline et al., 1951).

(2) Forme proliférative et destructive du sujet de plus de 30 ans (Généralement entre 40 et 50 ans). -Trente cas présentent des proliférations typiques analogues à celles considérées comme classiques. Vingt de ces cas sont sévères et l'atteinte très diffuse est généralement symétrique. Elle s'accompagne de raideur plus ou moins importante avec des déviations et des subluxations quelquefois très marquées.

Les radiographies révèlent dans ces cas sévères dont la durée d'évolution était pour quelques uns de 2 ou 3 ans seulement, des lésions destructives $\stackrel{2}{\overparen{D}}$ (Fig. 2) ou atrophiques (Fig. 4) extrèment impor- $\frac{}{\zeta}$ tantes. Les os du carpe présentent des contours $\square$ déchiquetés à l'emporte-pièce. Ces altérations sont très marquées à l'extrèmité inférieure du radius $\vec{F}$ et dans la région du carpe qui est en rapport avec $\stackrel{\text { ? }}{?}$ le deuxième métacarpien, plus particulièrement le응 trapèzoïde. Le carpe est généralement diminué de $\frac{\bar{\sigma}}{\bar{N}}$ hauteur; mais c'est surtout la distance radius- $\frac{\vec{\sigma}}{\sigma}$ deuxième métacarpien qui est le plus nettement $\varrho$ réduite, et il en résulte généralement une déviation क radiale du carpe plus ou moins marquée qui s'oppose $\vec{\circ}$ à une déviation cubitale des articulations métacarpo- $-\overrightarrow{-}$ phalangiennes.*

Les troubles de structure sont très marqués; il existe des zones de condensation qui bornent les? surfaces articulaires plus ou moins régulièrement $; \vec{A}$ il est intéressant de noter que la condensation et la destruction des surfaces articulaires sont concomitantes.

Le processus destructif peut soit être localisé à la Zone juxta-articulaire qui s'amenuise, et alors la destruc- $\square$ tion ne s'accompagne pas de condensation, soit s'étendre ou être localisé à la surface articulaire. Au niveau des phalanges et des metacarpiens la destruction des surfaces $\bar{\sigma}$ articulaires peut être le stade avancé de destructions qui® ont débuté dans la zone juxta-articulaire. Par contre, $\overrightarrow{0}$ au niveau d'autres articulations et en particulier \&s hanches, la destruction est d'emblée localisée à la surface? articulaire; il n'existe pas de destruction juxta-articulaiz. Les altérations radiologiques sont donc différentes suivant les articulations.

Il existe par ailleurs des phénomènes de lyse dans certains cas de polyarthrite dont nous ne parlons pas $\frac{}{\Phi}$ ici et que nous n'avons notés que chez la femme.

Or, pour les hanches, les zones de destruction correspondent aux zones d'appui normales puis $\supset$ pathologiques. Il nous parait en être de même pouro les poignets: destruction et condensation semblent être en rapport avec les zones ou s'exercent les pressions (Figs 2 et 5). Celles-ci sont: en partieo sous la dépendance de la contracture musculaire Les zones de condensation paraissent plus marquéesổ chez l'homme que chez la femme, alors que généralement l'ostéoporose, comme nous l'indiquons을 plus tard, est relativement discrète chez l'homme alors qu'elle est intense chez la femme. Outre을. ces zones de condensation, il existe des géodes soit ${ }_{\tilde{N}}$ dans le voisinage des zones détruites, soit dans les. épiphyses des doigts à la limite des pourtours $\tilde{O}$ articulaires. Ces géodes sont quelquefois difficiles à différencier de celles observées dans la goutte.

Ces altérations existent au niveau des autres articula tions et en particulier des hanches; elles sont intenses au

* Nous n'avons pas noté de dislocation du poignet, avec ou sans" destruction, dans nos cas de polyarthrite; celle-ci n'est pas rare dans $\square$ destruction, dans nos cas de polyarthrite; celle-ci n'est
les cas de polyarthrite chronique évolutive féminine. 


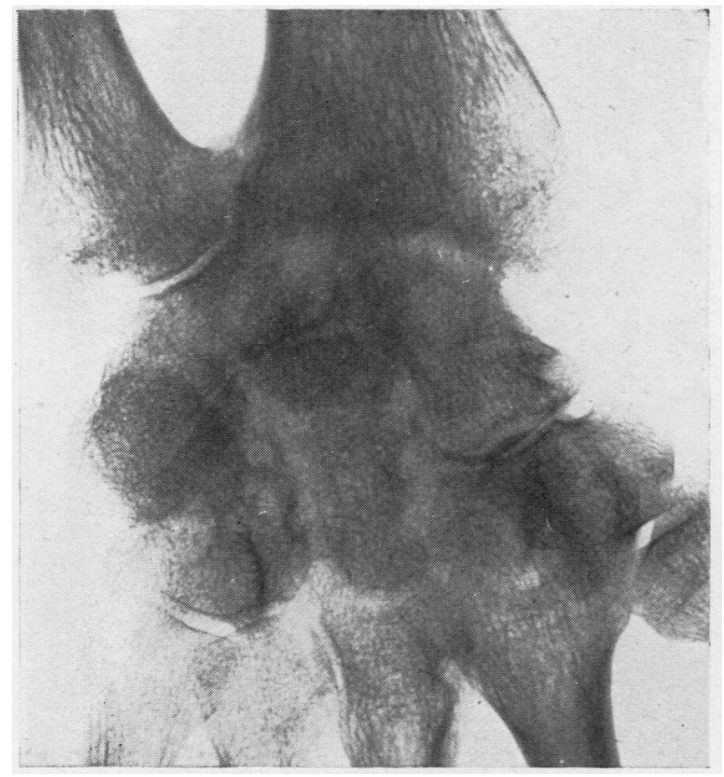

Fig. 1.-Polyartbrite de 9 ans d'évolution chez un homme de 24 ans. Atteinte des deux poignets à l'áge de 16 ans.

Le poignet droit est bloqué en extension. La palpation révèle un épaississement dur; le cliché montre une ankylose osseuse du carpe sans destructions. Les vestiges des surfaces articulaires indique que la hauteur des anciens interlignes n'a pas été modifiée. Aucun trouble de la structure.

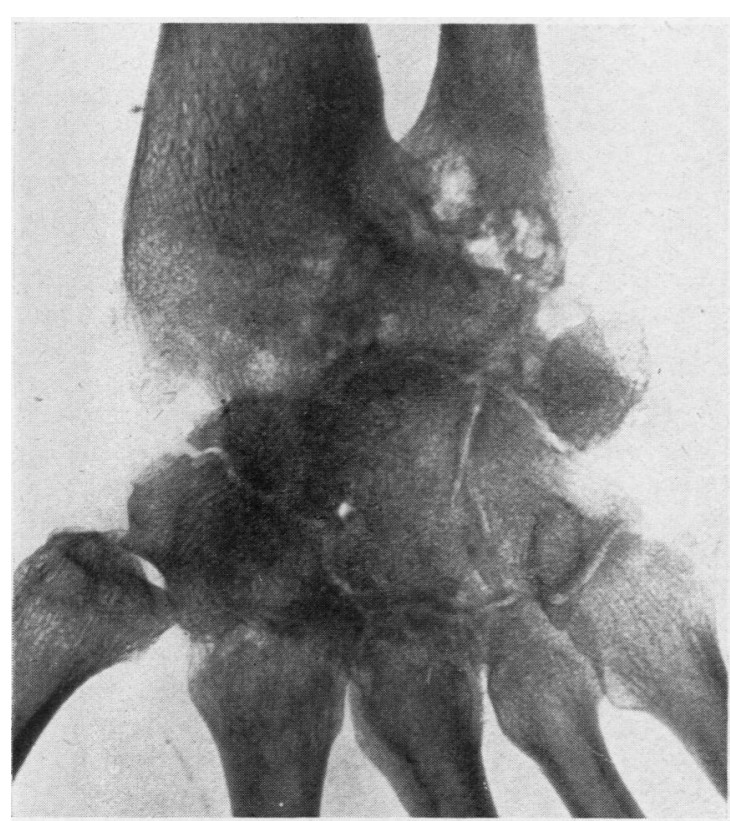

Fig. 3.-Polyarthrite de 40 ans d'évolution chez un homme de 55 ans. La polyarthrite a débuté à l'âge de 13 ans par petites poussées qui ont évolué pendant 10 ans. Puis le malade a présenté une nouvelle poussée vers 50 ans.

Le poignet gauche est ankylosé en rectitude; la radiographie révèle une ankylose de la première rangée du carpe avec le radius (ceci est à rapprocher de la Fig. 1) et des zones de condensation surtout importantes entre la première et la deuxième rangée des os du carpe. A noter des troubles importants de la structure, notammert à l'extrèmité inférieure du cubitus.

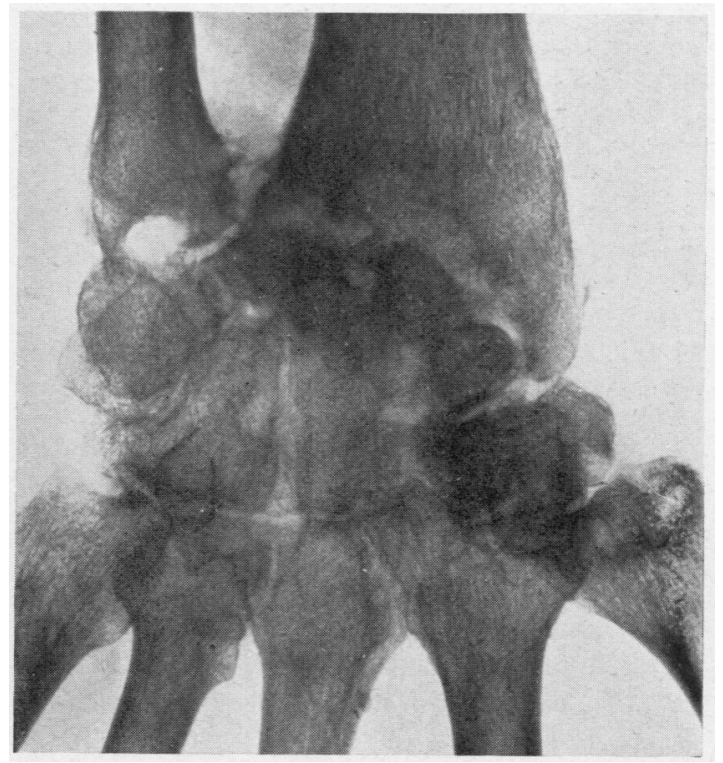

Fig. 2.-Polyarthrite masculine de 9 ans d'évolution chez un homme de 65 ans, ayant débuté à 56 ans. Forme sévère, très proliférative.

Poignet droit très enraidi: lésions destructives marquées, en particulier de la base du radius, aspect déchiqueté des contours. Les condensations sont importantes.

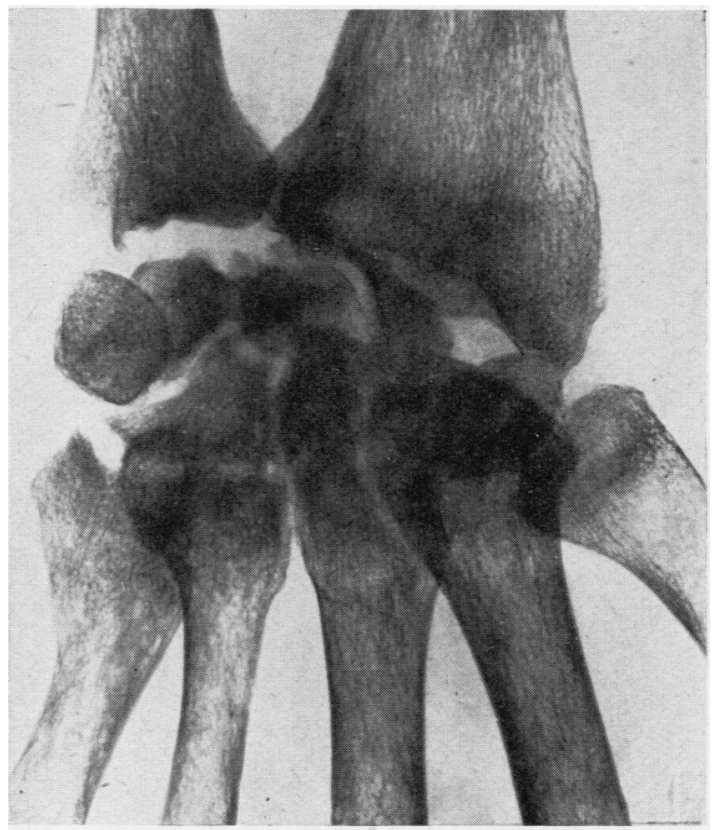

Fig. 4.-Polyarthrite sévère de 4 ans d'évolution chez un homme de 64 ans. Le poignet droit est très enraidi; il existe à ce niveau des proliférations molles et très douloureuses à la palpation. Le cliché révèle une atrophie extrèmement importante de tous les os du carpe; celui-ci est considérablement diminué de hauteur à tel point que la styloìde radiale est très près de la base du deuxième métacarpien. 
niveau des genoux alors que dans la polyarthrite féminine nous constatons une porose, un pincement, des déplacements, mais les destructions à ce niveau sont discrètes.

L'ostéoporose précède la destruction, le pincement articulaire est précoce. Les déplacements sont, dans cette forme, généralement en rapport avec les destructions qui sont d'apparition rapide. Mais dans quelques cas, les surfaces articulaires sont peu altérées mais les rapports de celles-ci très modifiés; ceci semble alors dû à une atteinte élective de l'appareil ligamentaire de maintien par le processus inflammatoire et une action prédominante des muscles fléchisseurs de la main par exemple.

(3) Forme hydarthrodiale survenant généralement à partir de 40-50 ans. - Dans sept cas il s'agit d'une atteinte diffuse des mains, mais pas toujours symétrique. Au niveau du poignet le gonflement articulaire se présente sous la forme de bosses plus ou moins marquées et il peut être opposé à l'empâtement diffus des proliférations. La palpation révèle qu'il s'agit de petits épanchements, et ceux-ci peuvent quelquefois être associés chez un même suiet à des proliférations; mais chez ceux de notre étude, il ne s'agissait que d'épanchements purs. La mobilité est conservée. La radiographie peut montrer soit un poignet normal soit une légère ostéoporose. Il est courant de remarquer que malgré l'importance de l'épanchement les déviations sont peu marquées. L'épanchement doit dilater les différents culs-de sacs synoviaux sans modifier l'appareil de maintien. De plus la douleur et la contraction musculaire sont moins prononcées.

Ces petits épanchements peuvent exister au niveau de toutes les articulations et, en particulier, des genoux. Dans cette forme, l'atteinte des hanches est extrèmement rare.

L'évolution de cette forme peut-être soit continue avec de petites poussées, soit, le plus souvent, intermittente. Cette forme, dont le pronostic nous paraît relativement bénin et dans laquelle la sédimentation est souvent élevée, est, d'autre part, très rebelle à la plupart des thérapeutiques.

" L'hydropisie polyarticulaire chronique", (Forestier et Certonciny, 1944; Forestier et al., 1949) touche surtout les grosses articulations et d'une manière symétrique: les mouvements sont peu limités sauf par le volume des épanchements. Il existe parfois de la laxité articulaire mais pour ainsi dire jamais d'attitudes vicieuses importantes.

Nous classons cette affection dans le cadre des rhumatismes inflammatoires mais nous ne savons si elle doit être distinguée de la polyarthrite surtout de la forme hydrarthrodiale. Les localisations sont certes différentes mais l'évolution de certains cas d'hydropisie poly-

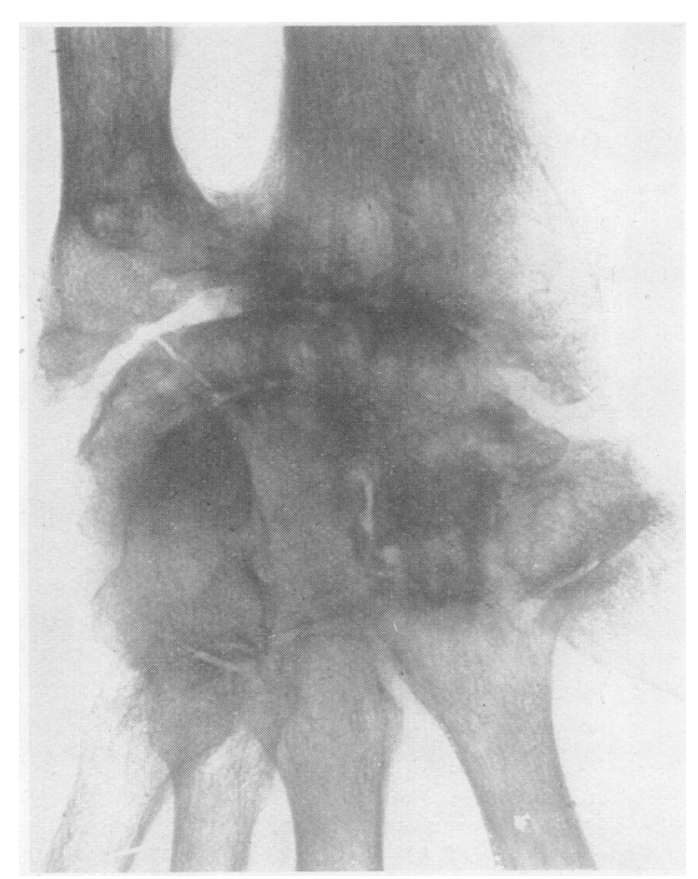

Fig. 5.-Polyarthrite de 27 ans d'évolution, chez un homme de 58 ans; cette polyarthrite sévère continue à évoluer.

Poignet droit bloqué: lésions destructives moins importantes @e ur la Fig. 2, ankylose osseuse de l'articulation radio-cubitigi inférieure non soumise à des pressions, déviation radiale du poigner.

articulaire suivis pendant de nombreuses années ont révèlé que cette affection pouvait évoluer vers une plus $\bar{\partial}$ grande diffusion, qu'il pouvait s'y associer plus ou moins tardivement des proliférations et des altérations des- $\stackrel{\mathbb{Q}}{\mathbb{Q}}$ tructives.

Les divers rhumatismes inflammatoires ne sont que des $\overline{\overline{0}}$ syndromes cliniques. Peut-être ne s'agit-il que de $\frac{3}{7}$ formes cliniques d'une seule maladie. (Nous mettons à part la spondylarthrite ankylosante.)

(4) Polyarthrite douloureuse du sujet de plus de 70 ans. - Deux de nos cas (nous rappelons que nous 3 . n'avons conservé pour ce travail que des dossiers complets) ont présenté un tableau de douleurs extrèmement vives, rapidement diffuses avec atteintes de l'état général-fièvre et amaigrissement. Dans ce tableau l'atteinte des hanches et surtout des $\frac{D}{0}$ épaules prédominait.* Actuellement, après un début bruyant qui date de 2 à 3 ans, l'affection ô semble se stabiliser. A l'examen: la douleur est $N$ vive à la palpation et à la mobilisation, mais il n'existe aucune prolifération synoviale, la radio- $\omega$ graphie ne révèle aucune altération, la raideur douloureuse des épaules semble surtout être la cause de l'invalidité relative de cas malades.

* Dans une prochaine publication, nous décrirons avec le Docteur A. Certonciny un tableau localisé aux articulations rhizoméliques. 
En résumé, l'âge du malade nous paraît jouer un rôle dans le type d'altérations radiologiques que nous pouvons constater au niveau des articulations où le processus rhumatismal a été sévère et persistant: chez l'homme jeune* la polyarthrite aboutit à une ankylose par transformation osseuse des éléments mous articulaires; chez l'homme de plus de 30 ans elle donne des destructions et des atrophies osseuses.

Cette ankylose du jeune pose un problème nosologique. La différence essentielle entre le type d'altérations radiologiques constatées au niveau des membres dans la spondylarthrite et celles constatées dans la polyarthrite était pour nous, que les premières, à leur stade avancé, étaient une ankylose et que celles de la polyarthrite étaient une destruction (Forestier et al., 1951). Or cette étude met en évidence que la polyarthrite du jeune peut aussi donner une ankylose osseuse sans destructions. $\dagger$

Néanmoins nous continuerons à considérer la polyarthrite et la spondylarthrite ankylosante comme deux entités cliniques différents. La spondylarthrite présente certains caractères cliniques (localisation rachidienne et surtout évolution ascendante, iritis, absence de nodules fibreux, même dans les cas avec atteinte des membres) et radiologiques (stade 1 et 2 des articulations sacro-iliaques, Forestier et Metzger, 1939, syndesmophytes ou ponts osseux juxta-discaux, absence d'altérations du type destructif dans les cas qui évoluent chez des sujets de plus de 30 ans), $\ddagger$ caractères qui lui appartiennent en propre. De plus, les localisations périphériques qui peuvent survenir au cours de la spondylarthrite sont différentes de celles de la polyarthrite: l'atteinte des mains et surtout des poignets et des coudes est extrèmement rare dans la spondylarthrite alors que l'atteinte des articulations acroméliques, même chez le jeune, est habituelle. L'ankylose chez le jeune s'observe donc sur des articulations différentes.

\section{Autres Notions Cliniques}

Des adénopathies furent notées dans huit cas (syndrome de Chauffard et Ramond), et des nodules fibreux dans neuf autres cas. Ceux-ci nous semblent donc plus frèquents que chez la femme.

\footnotetext{
* La polyarthrite de l'enfance donne aussi des ankyloses mais les épiphyses qui sont malléables en raison de l'âge et qui subissent en raison de la contracture musculaire des pressions importantes en raison de la contracture musculaire des pressions importantes
sont déformées. De plus, les attitudes vicieuses de la polyarthrite de l'enfance sont précoces et la pression s'exerce' dans des conditions anormales; ainsi se trouvent constituées des ankyloses osseuses avec des modifications importantes des rapports des épiphyses.

+ Il est même un type très particulier où le processus ankylosant est extensif, les articulations sacro-iliaques sont même atteintes mais il n'existe dans ces cas ni stade I et II des altérations atteintes mais de la classification as Forestier et juxta-discaux. Nous reprendrons sa description dans un prochain travail.

$\ddagger$ Les très rares cas de lyse que nous avons noté daus des cas de spondylartı hite au niveau du poignet ou de la hanche ou de malacie (colonne cervicale) sont survenus chez des sujets de plus de 50 ans.
}

Il existait une maladie de Dupuytren chez deux sujets présentant une polyarthrite.

Surtout nous avons noté dans sept cas, dont trois jeunes, des troubles trophiques importants: épaississement des téguments qui sont violacés et froids, alors que l'amincissement de la peau, phénomène fréquent chez la femme, n'a pas été constaté dans notre série. Les mains de nos malades peuvent évoquer dans certains cas le diagnostic de sclérodermie.

La polyarthrite de l'homme est sévère; sept de nos cas étaient grabataires, 31 ne pouvaient se déplacer qu'avec difficulté et ne pouvaient fournir un travail régulier; il s'agissait de deux sujets jeunes à forme ankylosante extensive et les autres plus âgés avec des proliférations et des destructions; les cas avec petits épanchements même multiples, n'étaient pas invalides.

De plus, la polyarthrite de l'homme semble plus rebelle aux diverses thérapeutiques que celle de la femme; et même les traitements hormonaux (cortisone et ACTH) paraissent soit donner des résultats moins spectaculaires, soit, après l'arrêt de ceux-ci, déclencher des poussées plus vives que chez la femme. La forme hydarthrodiale est, elle aussi, rebelle, mais, comme nous venons de l'indiquer, son pronostic est beaucoup plus favorable.

\section{Diagnostic Differentiel}

Le diagnostic ne se pose pratiquement qu'à la phase de monoarthrite ou d'oligo-arthrite. Dans le premier cas, s'il s'agit d'une atteinte persistante de l'épaule, le diagnostic de périarthrite non calcifiante ou adhésive ne doit pas être maintenu pendant plusieurs mois ou années; s'il s'agit d'une autre articulation périphérique, le diagnostic de monoarthrite infectieuse, surtout tuberculeuse, est généralement fait, et nous voyons trop de malades dont le diagnostic est suivi d'un acte chirurgical, alors qu'au moment de l'intervention il n'existait que des signes banaux inflammatoires: douleurs, gonflement, raideur, et légère ostéoporose. La preuve de l'infection tuberculeuse par la biopsie et par l'inoculation au cobaye doit être faite avant de décider une immobilisation plâtrée ou une résection des extrémités osseuses. Une irrégularité des surfaces articulaires nous paraît être extrèmement rare à la phase de monoarthrite d'un rhumatisme inflammatoire.

En présence d'atteinte pauci-articulaire de type inflammatoire, surtout si celle-ci est localisée aux membres inférieurs et en particulier aux pieds et aux chevilles, il faut éliminer: 
(1) La goutte polyarticulaire, qui est le diagnostic le plus difficile à éliminer; en effet, dans la goutte comme dans la polyarthrite, la localisation de début est souvent les pieds et les chevilles. Mais certains signes cliniques doivent permettre d'envisager le diagnostic de goutte: début et terminaison brutale de l'accès, localisation aux gros orteils. Par contre, une atteinte précoce d'un poignet ou d'une coude, exceptionnelle dans la goutte, oriente vers la polyarthrite. L'examen clinique doit comprendre la recherche des tophi sur les pavillons auriculaires, et des hygromas aux coudes. La radiographie peut révéler soit d'importantes géodes souscorticales de la goutte, soit de petites géodes que l'on ne peut distinguer de celles de la polyarthrite. C'est bien souvent le dosage de l'acide urique sanguin qui confirmera ou infirmera le diagnostic de goutte.

(2) La spondylarthrite ankylosante à début par localisation au niveau des membres: l'examen radiologique systèmatique des articulations sacro-iliaques doit être fait. Nous n'avons voulu retenir pour notre étude que les sujets ayant un dossier radiologique suffisant et une uricémie.

\section{Conclusions}

Il nous semble se dégager de l'étude de 48 dossiers cliniques et radiologiques de polyarthrite masculine quelques notions:

(1) L'âge joue un rôle dans le type d'altérations radiologiques avancées; images d'ankylose osseuse chez le jeune du même type que celles que l'on rencontre dans la spondylarthrite; images de destruction ou d'atrophie osseuse, associées ou non à des condensations, chez le sujet de plus de 30 ans.

(2) L'élément articulaire inflammatoire nous paraît variable aussi suivant l'âge: gonflement plus ou moins dur, sorte de blindage chez le jeune; proliférations synoviales chez le sujet de plus de 30 ans; douleurs simples chez le vieillard. L'épanchement plus ou moins abondant est surtout noté chez l'adulte de plus de 30 ans et ce n'est que chez lui que l'on rencontre les petits épanchements multiples réalisant la forme hydrarthrodiale.

(3) Il semble exister une relation entre l'élément inflammatoire et l'altération radiologique qui apparaît lors de la persistance de l'élément inflammatoire; la prolifération s'accompagne alors de destruction, l'épanchement d'une ostéoporose, l'épaississement dur ou blindage, d'ankylose osseuse.

Ce travail est basé sur un petit nombre de cas et il devrait être confirmé par un travail plus important, mais il nous a paru intéressant de mettre déjà en évidence l'importance de la notion d'âge; le terrain joue donc un rôle.

L'étude des altérations radiologiques suivant l'âge devra être faite chez la femme; mais dès maintenant nous pouvons dire que les phénomènes d'ankylose, sans destructions osseuses, n'apparaissent que chez l'adolescente ou la jeune adulte. Notre documentation ne nous permet pas de dire si les phénomènes destructifs ou atrophiques n'apparaissent unique $\frac{\square}{\omega}$. ment qu'à partir de l'âge de 30 ans.

De plus il est necessaire d'avoir des clichés radio $\overrightarrow{\vec{s}}$ graphiques de toutes les articulations aux diverso stades de leur évolution car, comme nous y avonso fait allusion plus haut, les altérations diffèrent产 suivant les localisations.

\section{BIBLIOGRAPHIE}

Barcelo Torrent, P. (1947). " Reumatismos articulares crónicos," 2ème édition, p. 60 . Salvat, Barcelona.

Collins, D. H. (1949). "The Pathology of Articular and Spinat $\vec{\omega}$ Diseases," p. 169. Arnold, London.

Fletcher, D. E., et Rowley, K. A.'(1952). Brit. J. Radiol., 25, 282.

Forestier, J., et Metzger, J. (1939). Presse méd., 47, 1247.

- et Certonciny, A. (1944),Arch. Rhum., p. 28. chronique," Communication à la IVe Conférence Scientifique. chronique," Communication à la IVe Conférence Scientifique
Internationale de Dax. Internationale de Dax.
Jacqueline, F., et Rotes-Quérol, J. (1951). “ La spondyli arthrite ankylosante," pp. 116 et 140 . Masson, Paris. O Françon, F. (1946). Conférences cliniques de rhumatologie pratique, Ière serie, $p .126$.

Herbert, J. J. (1951). Rev. Rhum., 18, 369.

Jacqueline, F. (1952). J. Radiol. Électrol., 33, 219.

- Depraitère, R., et Truet, J. (1951). Rev. Rhum., 18, 527. et Rebeyrotte (1952). Recherches sérologiques dans les rhumatismes chroniques inflammatoires. Réactions Communication à la Ligue contre le Rhumatisme.

Steindler, A. (1951). J. Bone Jt Surg., 33, 849.

\section{Rheumatoid Arthritis in the Male}

\section{SUMMARY}

Records of 48 male patients with rheumatoid arthritts were investigated. They comprised only cases where full clinical and radiological data, including $x$ rays of theô sacro-iliac jọints (to exclude ankylosing spondylitis) and of at least one affected joint, were available. The duration of the disease varied from a few months to 45 years. $\overrightarrow{\overrightarrow{0}}$ In nine cases the patients were followed-up for long 3 periods, a few of them for over 15 years.

Incidence.-The proportion in the male is given aso. 20 per cent., but only polyarticular symmetrical arthritis was considered.

Antecedents.-A precipitating factor, such as focalo infection, articular trauma, fracture of the skull, and 3 . cerebral stroke, was elicited in eleven cases.

Age of Onset.-In five cases the disease began between the ages of 16-20, in 28 cases between $30-50$, and in one case after the age of 70 .

\section{Early Symptoms}

(i) Local.-A monarticular onset was seen in six cases,, $0 \mathrm{~J}$ this single localization persisting for periods ranging from a few months to 6 years. In thirty cases with onset in two to four joints only, progress towards a typicakw symmetrical polyarthritis was slow (31 years in one case). Initial polyarthritis was seen in ten cases, in half of whicheo the manifestations were intermittent.

(ii) General.- The high incidence of general symptoms in the male is stressed. In 32 cases out of 48 the onset was marked by fever, loss of weight, or both. 


\section{Clinical and Radiological Changes}

This section is primarily concerned with clinical and radiological changes in the wrist joint. In the light of the observed relationship between morbid manifestations and the age of the patient, the material has been classified as follows:

(a) Ankylosis in the young adult without bone destruction. This form was seen in six patients aged 18-26 years. Clinically there was stiffness and sometimes locking of the joints, muscular atrophy masked by a hard thickening of the skin, which was pale and cold, and induration of the finger joints, but little deformity. $X$ rays showed ankylosis without destruction or structural changes.

(b) Proliferation and destruction in subjects over 30.Thirty cases (mostly between the ages of 40-50) exhibited the typical proliferative, destructive, and atrophic manifestations of established rheumatoid arthritis. It is stressed that such manifestations were observed also in cases of 2-3 years' duration only, contrasting with the non-destructive changes in the young adult.

(c) Effusion usually in the age group 40-50.-Here synovial effusions dominated the picture, while other symptoms such as osteoporosis, deformity, pain, and functional impairment were either absent or slight.

(d) Painful polyarthritis in subjects over 70.-In two elderly patients, the joints-mainly hip and shoulderwere extremely painful and tender, and there was fever and loss of weight. No radiological changes were observed.

\section{Other Clinical Observations}

Adenopathy was noted in eight cases. Nodules were seen in nine cases; they seemed to be more frequent in men than in women. Dupuytren's contracture existed in two cases.

Of particular interest were marked trophic changes of the skin, often simulating scleroderma; these were seen in seven patients, three of them young. Thinning of the skin, frequently seen in female arthritis, was not found in this series.

Polyarthritis in the male is a severe and painful affection; seven cases were bedridden, and in 31 functional impairment made regular work impossible; among the latter were two young men with extensive ankylosis. Furthermore, rheumatoid arthritis in the male is less responsive to treatment.

\section{Differential Diagnosis}

At the mono-arthritic stage the possibility of scapular peri-arthritis and of infectious, particularly tuberculous, arthritis should be considered.

In cases of oligo-arthritis the absence of tophi and of uricaemia will exclude gout. To avoid confusion with ankylosing spondylitis commencing in the limbs, an $x$ ray of the sacro-iliac joints is necessary.

\section{Conclusions}

The authors stress the importance of age as a factor determining the type of lesions: ankylosis in the young, synovial proliferation and bone destruction in the over30 s, pain in the old. The over-30 age group is more prone to suffer from synovial effusion and they alone may be subject to small multiple effusions. The relationship between the inflammatory elements and the radiological changes should be noted: proliferation is accompanied by destruction, effusion by osteoporosis, and hard thickening by bony ankylosis.

\section{Poliartritis masculina \\ CONCLUSIONES}

Del estudio de los datos clínicos y radiológicos de 48 casos de poliartritis masculina parecen emanar algunas nociones:

(1) La edad juega un papel en el tipo de alteraciones radiológicas avanzadas; imágenes de anquilosis ósea en el joven del mismo tipo que las que se encuentra en la espondilartritis; imágenes de destrucción o de atrofia ósea, a veces asociadas con condensaciones, en el sujeto de más de 30 años.

(2) El elemento articular inflamatorio también nos parece variable según la edad: tumefacción más o menos dura, especie de coraza, en el joven; proliferaciones sinoviales en el sujeto de más de 30 años; dolores simples en el viejo. El derrame más o menos abundante se nota en el adulto de más de 30 años y en él sólo se encuentra los pequeños derrames multiples que realizan la forma hidartrodial.

(3) Parece que existe una relación entre el elemento inflamatorio y la alteración radiológica que sobreviene al persistir el elemento inflamatorio; la proliferación acompáñase entonces de destrucción, el derrame de una osteoporosis, endurecimiento o coraza de anquilosis ósea.

Este trabajo se basa en un pequeño número de casos y precisa confirmación por un trabajo más importante, pero nos parece interesante exponer ahora la importancia de la noción de edad; el terreno juega pues un papel. 